\title{
CrashTest: A Fast High-Fidelity FPGA-Based Resiliency Analysis Framework
}

\author{
Andrea Pellegrini, Kypros Constantinides, Dan Zhang, Shobana Sudhakar, \\ Valeria Bertacco and Todd Austin \\ University of Michigan \\ \{apellegrini, kypros, danz, sshobana, valeria, austin\}@umich.edu
}

\begin{abstract}
Extreme scaling practices in silicon technology are quickly leading to integrated circuit components with limited reliability, where phenomena such as early-transistor failures, gate-oxide wearout, and transient faults are becoming increasingly common. In order to overcome these issues and develop robust design techniques for large-market silicon ICs, it is necessary to rely on accurate failure analysis frameworks which enable design houses to faithfully evaluate both the impact of a wide range of potential failures and the ability of candidate reliable mechanisms to overcome them. Unfortunately, while failure rates are already growing beyond economically viable limits, no fault analysis framework is yet available that is both accurate and can operate on a complex integrated system.

To address this void, we present CrashTest, a fast, highfidelity and flexible resiliency analysis system. Given a hardware description model of the design under analysis, CrashTest is capable of orchestrating and performing a comprehensive design resiliency analysis by examining how the design reacts to faults while running software applications. Upon completion, CrashTest provides a high-fidelity analysis report obtained by performing a fault injection campaign at the gate-level netlist of the design. The fault injection and analysis process is significantly accelerated by the use of an FPGA hardware emulation platform. We conducted experimental evaluations on a range of systems, including a complex LEON-based systemon-chip, and evaluated the impact of gate-level injected faults at the system level. We found that CrashTest is 16-90x faster than an equivalent software-based framework, when analyzing designs through direct primary I/Os. As shown by our LEONbased SoC experiments, CrashTest exhibits emulation speeds that are six orders of magnitude faster than simulation.
\end{abstract}

\section{INTRODUCTION}

As silicon process technology pushes towards smaller technology sizes, device reliability is an emerging challenge for next-generation designs [5]. Silicon failure mechanisms such as early transistor failures, gate-oxide wear-out, manufacturing defects, and radiation-induced soft errors threaten the design's reliability and severely reduce the yield and lifetime of future systems [20].

These reliability challenges are usually addressed either by conservative high-margin design techniques that avoid the manifestation of device failures during the product lifetime or by fault-tolerant techniques that detect failures and repair the system functionality in the field during operation [19]. Classic high-margin techniques for guaranteeing system reliability are rendered inadequate by extreme technology scaling and process variation [4]. The result is a steady shift towards the adoption of fault-tolerant techniques into the design flow of modern computing systems.

Recently, a number of commercial microprocessors that employ fault-tolerant design techniques have appeared in the marketplace $[10,15]$. Furthermore, the research area of fault- tolerant design is a well studied area and several solutions have been proposed in the literature $[2,6,19]$.

The need for Resiliency Analysis tools - Early in the design flow, system engineers need to assess the threats and reliability requirements of their design by employing resiliency analysis tools. They first gauge the robustness of the bare unprotected design to check if it meets the specified reliability target. If it does not meet the reliability target, a spectrum of fault-tolerant techniques must be considered and evaluated to select the ones that meet the target with the best trade-offs in implementation costs.

The process of accurately assessing the robustness of a bare unprotected design, or evaluating the effectiveness of candidate fault-tolerant techniques places the following requirements on the resiliency analysis infrastructure:

Low-level Fault Analysis: High fidelity is a very important aspect of the resiliency analysis framework. Using highlevel models of micro-architectural components with limited knowledge of the underlying circuit is inadequate to perform high-fidelity resiliency analysis. In order to correctly model the introduction, propagation, and possible masking of the faults, the resiliency analysis framework must accurately gauge circuit-level phenomena using a detailed low-level model of the design under analysis (e.g., gate-level netlist). Flexible Fault Modeling: Due to the existence of multiple silicon reliability threats, the resiliency analysis framework needs to support an extensive collection of low-level fault models to cover silicon failure mechanisms that range from transient faults, to manufacturing faults, process variation induced faults, and silicon wear-out related faults. Moreover, silicon fault modeling is an open area of research with continuous advancements [7,11]. Often, new fault models are devised targeting emerging silicon failure modes or more accurately modeling existing failure mechanisms. Therefore, it is crucial that an analysis framework's existing fault model collection could be easily upgraded with new fault models. Fast Design Simulation: The simulation of the design must deliver sufficient performance to enable the analysis of complex systems, including booting an operating system and run applications. This will allow users to assess the impact of faults at the full-system level, and still deliver evaluations with a fast turnaround.

Flexible Simulation Interface: It is critical for the usability of the framework to provide an intuitive way to analyze a wide range of designs and fault-tolerant techniques. Thus, a resiliency analysis framework demands a flexible interface and proper stubs to accommodate the evaluation of different systems. Given this challenging set of requirements 
for resiliency analysis, we focused our efforts toward the use of fault injection campaigns performed on gate-level models and accelerated by FPGA-based hardware emulation to achieve both accuracy and performance.

\section{A. Contributions of this Work}

In this work we present CrashTest, a novel resiliency analysis framework that addresses the challenges discussed above. We achieve this through the following contributions:

- We propose a new method to automatically orchestrate a fault injection campaign and perform a detailed fault monitoring and analysis on the gate-level netlist of the design. Our analysis approach accurately assesses the impact of run-time injected faults on the operation of a large complex system. The faults are injected using novel gate-level logic transformations that instrument the design's netlist with fault emulation logic.

- Our framework is augmented with a rich collection of fault models that encompass many types of faults that can be encounter at run time, ranging from soft faults to permanent silicon defects. These fault models are defined by different logic netlist transformations that can be easily modified and adapted by the user to model new failure mechanisms.

- We employed FPGA-based hardware emulation that enables the analysis of complex full-system designs that can boot an operating system and run applications. To the best of our knowledge, this is the first work that performs gate-level fault injection on a full-system design and observes the impact of the injected faults at the system level.

- We demonstrate the flexibility of our resiliency analysis framework by analyzing and presenting results for three complex designs including the LEON3 system-on-chip.

The remaining of the paper is organized as follows: Section II describes related work; Section III gives a highlevel overview of our framework; Sections IV and V explain in detail our injection methodology and the FPGAbased fault emulation techniques used by our framework, called CrashTest. Section VI evaluates the performance of CrashTest and presents experimental results that demonstrate its effectiveness. Finally, Section VII provides conclusions and directions for future work.

\section{RELATED WORK}

Fault Simulation vs. Resiliency Analysis - Fault simulators are software tools that determine the set of faults which can be exposed by a given test vector, and they are mainly used for ATPG (Automatic Test Pattern Generation) with the objective of measuring the coverage of a given set of test vectors [7]. On the other hand, resiliency analysis tools employ fault injection campaigns on a design executing typical workloads to measure the impact that the injected faults have on the design's operation and on the applications running on it.

Even though both methodologies use fault models to simulate the effects of faults on the circuit under test, their goals and requirements are fundamentally different. To prove the effectiveness of a given set of test vectors, fault simulators need to exercise faults in every node of the design for a limited amount of time. In contrast, resiliency analysis tools need to simulate the design under analysis for a significant amount of clock cycles in order to observe possible fault effects at the application level. Moreover, resiliency analysis tools usually employ Monte Carlo simulation methodologies and inject only the number of faults required to provide adequate statistical confidence in the results obtained. Due to these major differences, ATPG fault simulators cannot be practically used as a fault injection substrate to perform design resiliency analysis.

Several works in literature have proposed resiliency analysis frameworks that are based on fault injection. These works can be partitioned into software-based and hardware-based resiliency analysis, depending on the methodology used to perform the simulation and analysis [16].

Software-Based Resiliency Analysis - Often, softwarebased fault injection is preferred to hardware-based solutions due to its low cost, less complex development cycle, flexibility of customization, or simply because no low-level hardware model of the design is available. Several softwarebased resiliency analysis frameworks have been presented in literature $[12,17,21]$. Although they have many advantages, the speed of low level (e.g., gate-level) simulations does not make them viable solutions for analyzing complex designs or complete systems running applications.

Hardware-Based Resiliency Analysis - The performance limitation of the software-based fault injection approach can be addressed by employing hardware-based fault injection. Hardware-based resiliency analysis frameworks usually employ FPGAs (Field Programmable Gate Arrays), capable of emulating the fault-injected design orders of magnitude faster than software-based approaches, therefore significantly speeding up the process. Although the use of FPGA emulation platforms addresses the limited performance of the software frameworks, it introduces some major challenges in automating the fault injection and the analysis process. Furthermore, it is difficult to map complex fault models on the FPGA fabric. Hence, the hardware-based resiliency analysis frameworks proposed so far are limited to simple transient fault models and stuck-at faults [9, 14].

\section{FRAMEWORK OVERVIEW}

The goal of our resiliency analysis framework is to provide a fast, high-fidelity and comprehensive analysis of the effects of a broad range of fault classes on the applications running on a design under analysis (this could be either an unprotected design or a fault-tolerant design).

Given the specification of the design under analysis in a hardware description language (HDL), CrashTest automatically orchestrates a fault injection/analysis campaign. This process is composed of two stages: (i) a front-end translation that generates the fault-injection ready gate-level netlist for the design, and (ii) a back-end fault simulation and analysis that performs the actual fault injection and fault monitoring, 


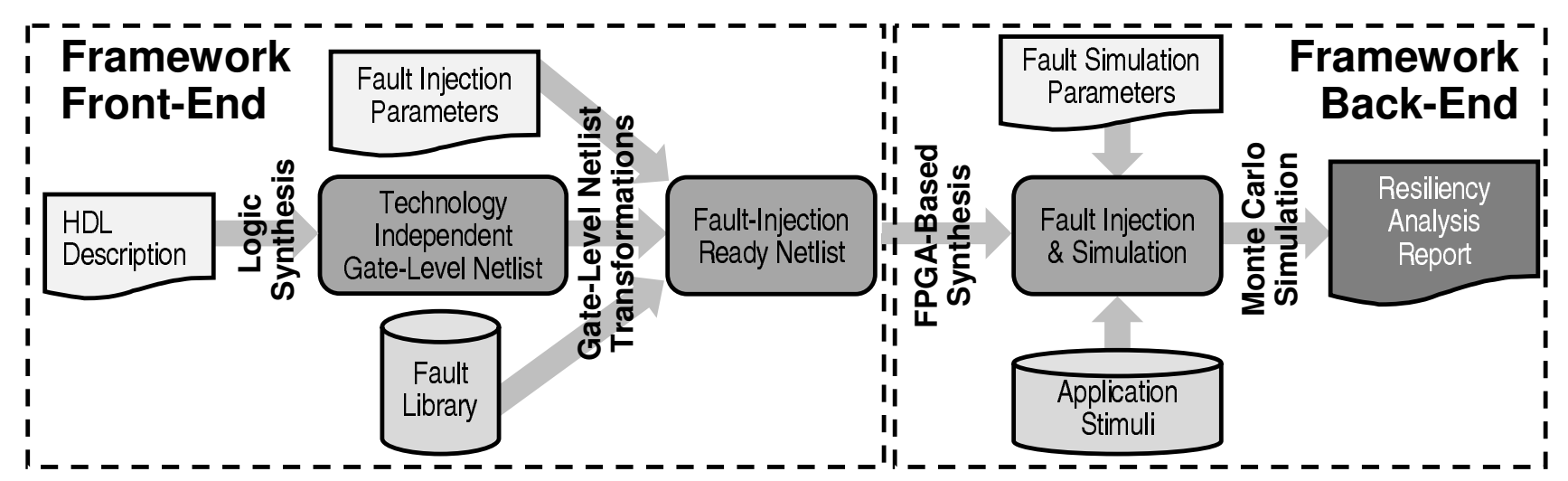

Fig. 1. Overview of the resiliency analysis framework: The framework is composed of (i) the front-end stage generating the fault injection-ready gate-level netlist and (ii) the back-end stage performing fault injection and analysis and generating the final resiliency analysis report.

and evaluates the effects of the injected faults. Figure 1 represents an overview Crashtest.

Only a FPGA solution can provide sufficient performance to run software applications on large and complex designs. However, a major cost in adopting this solution is the overhead necessary to map a design on the FPGA fabric. For complex designs the time required to generate a netlist mappable on the FPGA device can be prohibitively long. To reduce this overhead, in Crashtest we insert multiple faults in a same mapped netlist. These faults can be dynamically activated individually via software, amortizing the synthesis cost over several analyses.

Framework Front-End - First, on HDL model of the design under analysis is synthesized by the front-end stage of the framework using a standard cell library to obtain a gate-level netlist. CrashTest does not require the use of any particular library, as long as the chosen library can be properly modified to support the fault models.

For each standard cell in the target library (i.e., a combinational gate or a sequential element), CrashTest provides a gate-level logic transformation that modifies the cell and inserts extra fault injection logic. This extra logic can be activated at runtime to emulate the effects of a fault injected into the cell. We developed a wide range of fault models and gate-level logic transformations to provide the capability of emulating different failure mechanisms. The collection of all logic transformations is stored in the framework's fault library. Based on the injection parameters selected by the user (i.e., the fault models and the injection locations), the framework automatically generates the fault injection-ready netlist selecting the transformed cells in the library. This netlist is then transferred to the fault analysis simulator.

Framework Back-End - At the framework back end, the fault injection-ready netlist is re-synthesized to target an FPGA. At this point the fault injection and analysis campaign is ready to begin. Based on the fault simulation parameters given by the user, the fault injection/analysis emulator injects faults at different sites in the netlist and monitors their propagation and impact on the design and the running applications. During fault emulation, the design under analysis is exercised with the application stimuli. To gain statistical confidence on the provided results, the experiments are repeated in a Monte Carlo simulation model by altering the fault sites and/or the application stimuli. After running a sufficient number of experiments to gain statistical confidence, the results are aggregated into the resiliency analysis report which is the final deliverable of the CrashTest framework. In the following sections, we describe each step of the CrashTest framework in more detail.

\section{Gate-Level Fault Injection Methodology}

Technology Independent Logic Synthesis - The first step in the front-end stage of the CrashTest framework is to convert the user-provided high-level HDL model of the design under analysis into a common format that the framework can analyze to obtain an accurate list of candidate circuit locations for fault injection. This is achieved through logic synthesis with Synopsys Design Compiler targeting a technology-independent standard cell library (GTECH). The resulting gate-level netlist is composed of simple logic gates (e.g., AND, OR, NOT, Flip-Flops, etc, ) and it is free from any fabrication technology-related characteristics. This netlist is subsequently parsed to generate a list of all possible injection locations, that is, a list of all logic gates and flipflops in the design. This list can be used to specify the injection locations; alternatively, if randomized fault injection is desired, a random selection of fault sites can be performed by the framework.

Netlist Fault Injection Instrumentation - After fault locations selection, the gate-level netlist is instrumented with extra fault injection logic that, when enabled, emulates the effects of the injected faults. Each fault model supported by the framework is associated with a specific gate-level logic transformation to achieve this goal. The collection of gatelevel logic transformations composes the framework's fault library. This modular design makes a straight forward task upgrading the framework with new fault models by simply implementing new logic transformations into the fault library. Our resiliency analysis framework is already equipped with a large collection of fault models and their corresponding netlist logic transformations. This collection spans an extensive spectrum of silicon failure mechanisms, ranging from transient faults due to cosmic rays, to permanent faults due to silicon wearout. Table 1 shows a list of supported fault models along with a brief description. 
Transistor-level NAND2 gate

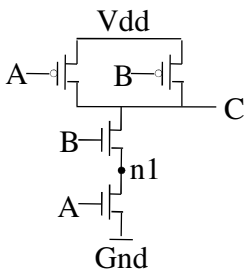

(a)
Fault Symptom Table

\begin{tabular}{|l|c|c|c|c|}
\hline A & 0 & 1 & 0 & 1 \\
\hline B & 0 & 0 & 1 & 1 \\
\hline Fault-Free & 1 & 1 & 1 & 0 \\
\hline Bridge-A-B & 1 & X & X & 0 \\
\hline Bridge-A-C & X & 1 & X & X \\
\hline Bridge-A-n1 & 1 & 1 & 1 & X \\
\hline Bridge-B-n1 & 1 & $X$ & 1 & X \\
\hline Bridge-B-C & X & X & 1 & X \\
\hline Bridge-C-n1 & 1 & 1 & $X$ & 0 \\
\hline
\end{tabular}

(b)
Instrumentation logic for Bridge-A-B

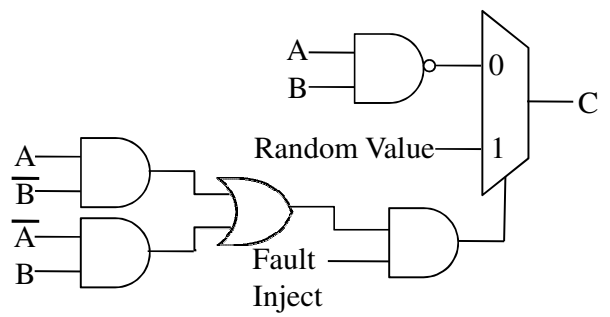

(c)

Fig. 2. Logic Transformations - Bridge Fault: The CMOS transistor-level design of a gate in (a) is used to generate the gate's fault symptom table for the fault model shown in (b). Part (c) shows the instrumentation logic for emulating the effects of the Bridge-A-B fault.

\begin{abstract}
Stuck-at: The stuck-at fault model is the industry standard model for circuit testing. It assumes that the defect behaves as a node stuck at logical 0 or 1 . The stuck-at fault model is most commonly used to mimic permanent manufacturing or wearout-related silicon defects.

Stuck-open: The stuck-open fault model assumes that a single physical line in the circuit is broken. The unconnected node is not tied to either Vcc or Gnd and its behavior is rather unpredictable (logical 0 or 1 or high impedance). This model is commonly used to mimic permanent defects that are not covered by the stuck-at fault model.
\end{abstract}

Bridge: The bridge fault model assumes that two nodes of a circuit are shorted together. The behavior of the two shorted nodes depends on the values and strength of their driving nodes. This model covers a large fraction of permanent manufacturing or wearout-related defects.

Path-delay: The path-delay fault model assumes that the logic function of the circuit is correct, but that the total delay in a path from inputs to outputs exceeds the allocated threshold and causes incorrect behavior. This model is used to mimic the effects of process variation or device degradation due to age-related wearout.

Single Event Upset: The single event upset (SEU) fault model assumes that the value of a node in the circuit if flipped for one cycle. After this one-cycle upset, the node returns to its normal behavior. The SEU fault model is used to mimic transient faults commonly caused by cosmic radiation or alpha particles.

Table 1. Fault models: CrashTest is enhanced with an extensive collection of fault models. These fault models cover transient faults as single event upsets and also a variety of permanent hard faults related to manufacturing, wearout, and process variation silicon defects.

Gate-Level Logic Transformations - Some fault models require trivial gate-level logic transformations. For example, the instrumentation needed to emulate a stuck-at fault is just a multiplexer that controls the output of the faulty gate and has one of its inputs connected to logic zero/one. However, there are more complex fault models which affect the design at the transistor level. For example, the bridge fault model assumes that two circuit nodes in the design are shorted together. To emulate the effect of a bridge model with high fidelity, we simulated the faulty CMOS gates at the transistor level and generated a corresponding fault symptom table. To illustrate this process with an example, Figure 2(a) shows the CMOS transistor level representation of a NAND2 logic gate, while Figure 2(b) shows the corresponding fault symptom table.

By observing the table we notice that the effects of the fault are masked for some input combinations, thus the faulty gate behaves as a fault-free gate. However, for other input

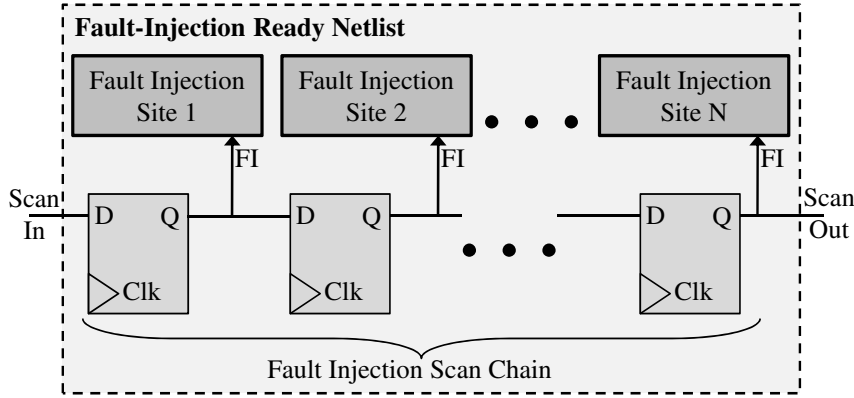

Fig. 3. Fault injection scan chain: The netlist is instrumented with fault injection logic for multiple faults. The scan chain controls the fault injection during emulation.

combinations the fault's effects propagate to the gate's output and result is an unstable output signal (Random Value in Figure 2(c)). The framework's fault library is populated with a fault symptom table for each combination of standard cell library gate and supported fault model. Given a gate type and a fault model, the instrumentation engine accesses the fault library and applies the corresponding logic transformation to instrument the fault. Figure 2(c) shows the instrumentation logic for a bridge fault between circuit nodes A and B of the NAND2 gate. A fault-tolerant design should be capable of handling these faults and either mask the errors introduced or reconfigure itself to exclude the faulty part of the design.

Path-Delay Fault Model - The logic transformations required by most fault models are similar to the one presented in Figure 2(c) for the bridge fault. One exception is the pathdelay fault model which has slightly different characteristics. Path-delay faults are characterized by slower combinational logic gates causing longer delays than foreseen at design time. Whenever these slower gates are exercised, they may cause timing violations (i.e., flip-flops at the end of the path miss to latch the newly computed value). In our framework, the effects of the path-delay fault model are emulated by a gate-level logic transformation such as that in Figure 4. To determinate the set of flip-flops affected by the slower faulty gate, we trace forward through the combinational logic and find all those flip-flops that have a path including the faulty gate. From that set of flip-flops we choose only those that have a path delay with a timing slack smaller than a predefined threshold specified by the user (i.e., the expected 


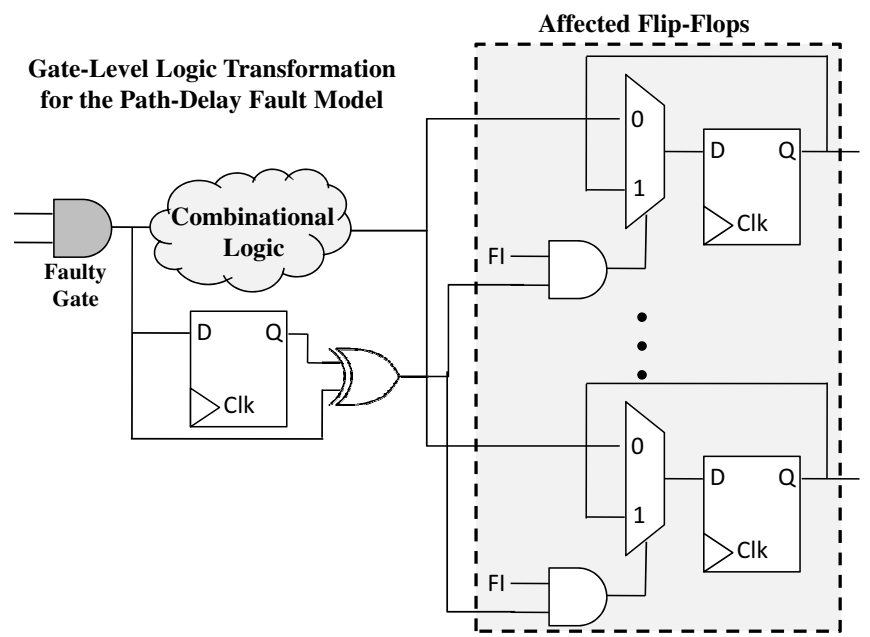

Fig. 4. Logic transformation for the path-delay fault model: If the output of the faulty gate changes in a given cycle, all affected flip-flops miss latching the newly computed value and hold the previous cycle's value.

delay due to the faulty gate).

Fault Injection Scan Chain - To avoid re-instrumenting the netlist each time a new fault must be injected, the netlist can be instrumented for several faults at multiple locations. This accelerates the emulation at the back-end of the framework, but also increases the instrumented circuit size. Moreover, the insertion of each fault into the netlist adds an extra control signal required for enabling and disabling it at runtime (for instance, signal Fault Inject and Random Value in 2(c)). As shown in Figure 3, these control signals are latched and connected to a scan chain, which at runtime are accessible by the Fault Injection Manager (see Figure 5).

\section{FPGA-BASEd FAult Emulation}

CrashTest employs an FPGA platform to emulate the fault injected hardware and accelerate the fault simulation and analysis process. The first step in this process is to synthesize and map the fault injection-ready netlist to the target FPGA. To provide a standard simulation interface that is independent of the design under analysis, we add an automatically generated interface wrapper to the fault injected-ready netlist. This interface wrapper provides a seamless connection with the fault injection manager, an automatically-generated software program responsible for orchestrating the fault injection and analysis campaign. The interface wrapper and the fault injection manager are connected through an on-chip interconnect bus. Figure 5 shows the major components and the data-flow of the fault injection, simulation and analysis process.

In our experiments we used a Xilinx Virtex-II Pro FPGA, which has two on-chip PowerPC processors, and run the fault injection manager software on one of them. Alternatively, the fault injection manager can also run on a soft-core mapped on the FPGA itself (e.g., Microblaze). The fault injection manager is responsible for several tasks:

1) Feed the instrumented injection scan-chain with all the control signals required in the fault injection campaign. This is done through a FIFO queue updated each time a new fault is activated into the design. The fault injection parameters (i.e., fault location and time) are stored on an off-chip memory accessible by the injection manager.

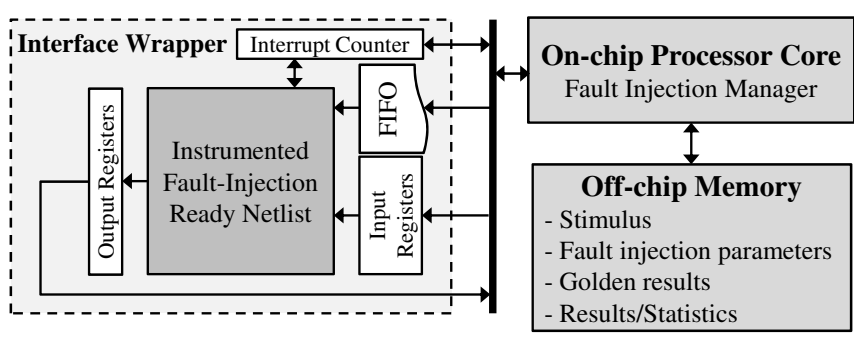

Fig. 5. FPGA-Based fault injection and simulation: The FPGA-mapped netlist is wrapped by a standard interface providing a seamless connection to the fault injection manager that is running on an on-chip processor core.

2) Stimulate the design through the input registers. The applications stimulus is either provided by the user or automatically generated, and it is stored in the off-chip memory. 3) Monitor the output of the FPGA-mapped design for errors through the output registers. The output is compared to a golden output that is collected with a fault-free version of the same design and it is stored in the off-chip memory.

4) Maintain fault analysis statistics and store the results to the off-chip memory for later processing.

5) Synchronize the FPGA-mapped design with the fault injection process through the interrupt counter.

\section{FrAMEWORK EVALUATION}

In this section, we evaluate our FPGA-based resiliency analysis infrastructure and compare its performance to an equivalent software-based implementation. We also perform an initial examination, using the CrashTest infrastructure, of the effects of different fault models in design resiliency.

\section{A. Experimental Methodology}

Benchmark Designs - For the evaluation of CrashTest we used three benchmark designs, described everywhere in Table 2. The chip-multiprocessor (CMP) interconnect router implements a wormhole router pipelined at the flit level with credit-based flow control functionality for a two-dimensional torus network topology [13]. We used SPEC CPU2000 communication traces derived from the TRIPS architecture [18] to provide application stimuli to the router. The DLX core is a 32-bit 5-stage in-order single-issue pipeline running the MIPS-Lite ISA. Finally, the LEON3 is a system-onchip including a 32-bit 7-stage pipelined processor running the SPARC V8 architecture, an on-chip interconnect, basic peripherals and a memory controller [10]. The LEON3 SoC is capable of booting an unmodified version of Linux 2.6. The LEON processor was configured without on-chip caches and faults were injected only in the core component.

Netlist Fault-Injection Instrumentation - The HDL model of the design under analysis is synthesized using Synopsys' Design Compiler and the GTECH standard cell library. The resulting netlist is a technology-independent GTECH gatelevel netlist. The gate-level netlist is then analyzed by Perl scripts to locate all possible injection sites in the circuit. Once the sites and fault types are selected (using a uniform random distribution for these experiments), a Perl script applies the required gate-level logic transformations.

Software-Based Analysis Methodology - The softwarebased fault simulation and analysis is performed using Synopsys' VCS logic simulator for the CMP router and the DLX 


\begin{tabular}{|l|c|c|l|}
\hline $\begin{array}{c}\text { Benchmark } \\
\text { Name }\end{array}$ & $\begin{array}{c}\text { Logic Gates } \\
\text { (GTECH) }\end{array}$ & $\begin{array}{c}\text { Flip } \\
\text { Flops }\end{array}$ & \multicolumn{1}{c|}{ Description } \\
\hline CMP Router & 16,544 & 1,705 & $\begin{array}{l}\text { chip-multiprocessor interconnect router for a } \\
\text { 2D mesh network with 32-bit flits }\end{array}$ \\
\hline DLX Core & 15,015 & 2,030 & $\begin{array}{l}\text { 5-stage in-order DLX pipeline running } \\
\text { MIPS-Lite ISA }\end{array}$ \\
\hline $\begin{array}{l}\text { LEON3 } \\
\text { System- } \\
\text { on-chip }\end{array}$ & 66,312 & 6,925 & $\begin{array}{l}\text { System-on-chip with a 7-stage pipeline 32-bit } \\
\text { processor compliant with the SPARC V8 } \\
\text { architecture, an on-chip interconnect, basic } \\
\text { peripherals and a memory controller. }\end{array}$ \\
\hline
\end{tabular}

Table 2. Benchmark Designs: Characteristics of the benchmark designs used to evaluate the CrashTest framework.

\begin{tabular}{|c|c|c|c|}
\hline Confidence Level = 95\% & \multicolumn{3}{|c|}{ Confidence Interval } \\
\hline $\begin{array}{c}\text { Number of Fault } \\
\text { Injections (Sample Size) }\end{array}$ & $\begin{array}{c}\text { CMP Router } \\
\text { (18249 gates) }\end{array}$ & $\begin{array}{c}\text { DLX Core } \\
\text { (17045 gates) }\end{array}$ & $\begin{array}{c}\text { LEON3 } \\
\text { (73237 gates) }\end{array}$ \\
\hline 256 & \pm 6.08 & \pm 6.08 & \pm 6.11 \\
\hline 512 & \pm 4.27 & \pm 4.27 & \pm 4.32 \\
\hline 1024 & \pm 2.98 & \pm 2.96 & \pm 3.04 \\
\hline 2048 & \pm 2.04 & \pm 2.03 & \pm 2.14 \\
\hline 4096 & \pm 1.35 & \pm 1.33 & \pm 1.49 \\
\hline 8192 & \pm 0.8 & \pm 0.78 & \pm 1.02 \\
\hline
\end{tabular}

Table 3. Statistical Confidence: The Table shows the confidence level of the results obtained when different number of faults are injected during the injection campaigns for our benchmark designs.

core. For the simulation of the LEON3 system-on-chip we used ModelSim, since it required the simulation of both Verilog and VHDL modules. The fault simulations using VCS were run on an Intel Core 2 Duo running at $2.13 \mathrm{GHz}$ with 2GB of RAM; while the ModelSim simulations were run on a $\mathrm{P} 4$ at $3.4 \mathrm{GHz}$ and $2 \mathrm{~GB}$ RAM.

FPGA-Based Analysis Methodology - For the FPGAbased fault emulation and analysis we used the XUP V2P Development Board [1]. The board is equipped with a Virtex2 Pro XC2VP30 FPGA with 13,696 slices (each with two 4-input LUTs and two flip-flops), and two PowerPC 405 processors. At the time of writing, this FPGA represented a mid-sized device; devices with up to ten times more resources are currently available. For off-chip memory we used one 256MB module of DRAM. The main tools used to map designs in the CrashTest framework are Xilinx Platform Studioand Synplicity's Synplify. The FPGA synthesis and mapping process was ran on a $44 \mathrm{CPU}$ at $3.0 \mathrm{Ghz}$ equipped with 1GB RAM. The synthesis and mapping process for the LEON3 system took about 45 minutes, while the other two benchmark designs required significantly less time.

\section{B. Monte Carlo Simulation \& Statistical Confidence}

Performing gate-level fault injection campaigns in complex designs and observing their impact at the application level is a computationally intensive process. The propagation of fault effects from the gate-level up to the application level requires a simulation of the design under analysis. To reduce the number of fault injections and make the resiliency analysis computationally tractable we adopted a Monte Carlo analysis method. Through Monte Carlo simulation, fault injection experiments are repeated several times, randomly changing the fault injection location and time (i.e., the clock cycle at which fault is enabled). The desired statistical confidence affects the number of repetitions of the experiment.

Table 3 shows the confidence intervals for different numbers of fault injection experiments for our three benchmark designs. These figures were calculated using the statistical

\begin{tabular}{|c|c|c|c|c|}
\hline $\begin{array}{l}\text { Bench. } \\
\text { Design }\end{array}$ & $\begin{array}{l}\text { Injected } \\
\text { Faults }\end{array}$ & $\begin{array}{c}\text { Slices } \\
\text { (out of 13696) }\end{array}$ & $\begin{array}{c}\text { Slice Flip } \\
\text { Flops } \\
\text { (out of 27392) }\end{array}$ & $\begin{array}{l}4 \text { Input LUTs } \\
\text { (out of 27392) }\end{array}$ \\
\hline \multirow{8}{*}{$\begin{array}{l}\text { CMP } \\
\text { Router }\end{array}$} & 0 (baseline) & $2968(21 \%)$ & $3021(11 \%)$ & $3705(13 \%)$ \\
\hline & 0 (wrapper) & $6679(48 \%)$ & $4731(17 \%)$ & $10840(39 \%)$ \\
\hline & 8 & $6718(49 \%)$ & $4745(17 \%)$ & $10781(39 \%)$ \\
\hline & 64 & $6912(50 \%)$ & $4857(17 \%)$ & $11192(40 \%)$ \\
\hline & 128 & $7161(52 \%)$ & $4985(18 \%)$ & $11408(41 \%)$ \\
\hline & 256 & $7279(53 \%)$ & $5241(19 \%)$ & $11425(41 \%)$ \\
\hline & 512 & $7854(57 \%)$ & $5753(21 \%)$ & $12020(43 \%)$ \\
\hline & 1024 & $8903(65 \%)$ & $6778(24 \%)$ & $13059(47 \%)$ \\
\hline \multirow{3}{*}{$\begin{array}{l}\text { DLX } \\
\text { Core }\end{array}$} & 0 (baseline) & $2499(18 \%)$ & $2520(9 \%)$ & $2386(8 \%)$ \\
\hline & 0 (wrapper) & $6820(49 \%)$ & $8202(29 \%)$ & $4573(16 \%)$ \\
\hline & 1024 & $9593(70 \%)$ & $6700(24 \%)$ & $9948(36 \%)$ \\
\hline \multirow{3}{*}{$\begin{array}{l}\text { LEON3 } \\
\text { System- } \\
\text { on-chip }\end{array}$} & 0 (baseline) & $10281(75 \%)$ & $10178(37 \%)$ & $20562(75 \%)$ \\
\hline & 0 (wrapper) & $11057(80 \%)$ & $11103(40 \%)$ & $22113(80 \%)$ \\
\hline & 1024 & $11785(86 \%)$ & $13146(47 \%)$ & $23570(86 \%)$ \\
\hline
\end{tabular}

Table 4. Fault injection logic overhead: Utilization of the FPGA resources comparing the baseline (fault-free) designs and the fault injection instrumented designs mapped on the FPGA.

sample size equations from [3]. We deemed that for most applications, a confidence level of $95 \%$ and a confidence interval of $3 \%$ are acceptable. From Table 3 we notice that this degree of statistical confidence can be achieved by just 1024 fault injections for all three benchmark designs.

\section{Framework Performance}

Fault Injection Logic Overhead - Table 4 shows the allocated FPGA resources when the baseline (fault-free) benchmark designs were synthesized and mapped on the FPGA. When the designs are augmented with the fault simulation interface wrapper, the utilization of the FPGA slices is increased from $15 \%$ to $31 \%$. As shown in the fourth and fifth columns of Table 4, not all of the flip-flops and LUTs in each utilized slice are used. The table also shows the overhead of the instrumentation logic for designs injected with different numbers of stuck-at faults. The capability of injecting several faults into the design is very important since it significantly accelerates the fault simulation process by avoiding time-consuming iterations of netlist instrumentation and FPGA synthesis/mapping.

Fault Simulation/Analysis Speed - Table 5 compares the speed of a software-based and our FPGA-based fault emulation and analysis engines. For the CMP router design we noticed that the speed of a software-based scheme varied for different fault models. This difference stems from the different logic complexity required to emulate the behavior of each fault model. On average, for the CMP router the software-based scheme provides a simulation speed that is on the order of $10 \mathrm{KHz}$. We have observed similar results for the DLX core design (not shown in the table for brevity). On the other hand, the speed of the FPGA-based scheme is not affected by the fault injection logic. Therefore, all fault models are emulated with the same clock frequency and achieve the same emulation speed. For the CMP router, the speed of the emulation framework is $220 \mathrm{KHz}$, leading to an average speed up of $\approx 20 \mathrm{X}$ for simple fault models and $\approx 85 \mathrm{X}$ for the more complex ones.

The simulation speed achieved by the software-based scheme when analyzing the LEON3 system-on-chip is much 


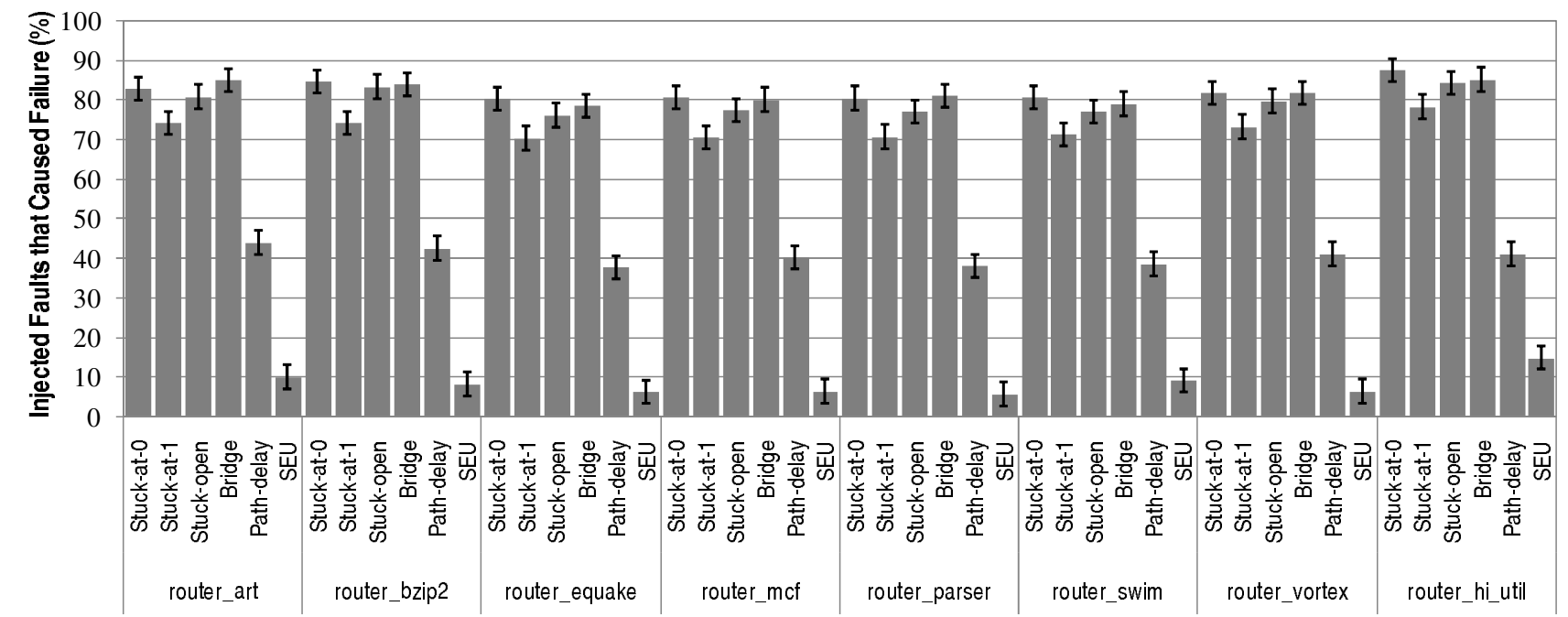

Fig. 6. Design Resiliency vs. Underlying Fault Model: Percentage of injected faults that were exposed for each fault model. Experiments are run on the CMP router using SPEC2000 traces.

\begin{tabular}{|c|c|c|c|c|}
\hline $\begin{array}{l}\text { Bench. } \\
\text { Design }\end{array}$ & $\begin{array}{l}\text { Fault } \\
\text { Model }\end{array}$ & $\begin{array}{c}\text { Software-Based Fault } \\
\text { Simulation Speed }\end{array}$ & $\begin{array}{l}\text { FPGA-Based Fault } \\
\text { Simulation Speed }\end{array}$ & Speed Up \\
\hline \multirow{6}{*}{$\begin{array}{l}\text { CMP } \\
\text { Router }\end{array}$} & Stuck-at-0 & $9.75 \mathrm{KHz}$ & \multirow{6}{*}{$220 \mathrm{KHz}$} & $22 X$ \\
\hline & Stuck-at-1 & $8.09 \mathrm{KHz}$ & & $27 X$ \\
\hline & Stuck-open & $2.42 \mathrm{KHz}$ & & $90 \mathrm{X}$ \\
\hline & Bridge & $2.63 \mathrm{KHz}$ & & $83 X$ \\
\hline & Path-delay & $11.34 \mathrm{KHz}$ & & $19 X$ \\
\hline & SEU & $13.04 \mathrm{KHz}$ & & $16 \mathrm{X}$ \\
\hline $\begin{array}{l}\text { LEON3 } \\
\text { System- } \\
\text { on-chip }\end{array}$ & Stuck-at-0 & $28 \mathrm{~Hz}$ & $25 \mathrm{Mhz}$ & $\sim 900000 \mathrm{X}$ \\
\hline
\end{tabular}

Table 5. Fault simulation speed: Performance comparison of the softwareand FPGA-based fault simulation engines.

lower than the one observed for the other two simpler designs (i.e., the CMP router and the DLX core). Specifically, the simulation speed is limited to $28 \mathrm{~Hz}$, due to the much higher complexity of the full-system LEON3 design. In contrast, the emulation speed of the LEON3 system on the FPGAbased scheme is faster than the other two simpler designs. This is due to how the application stimulus is applied to different designs by the fault injection manager. Since the LEON3 full-system design includes a memory controller, the interaction with the external environment is limited to memory read/write requests, which are served by the off-chip DRAM module. Therefore, in the LEON3 analysis there is very little interaction between the fault injection manager and the design under analysis in feeding the application stimulus. On the other hand, when emulating the other two designs, the fault injection manager must provide input stimuli cycle-bycycle in order to drive the emulation, thus limiting the overall performance. The emulation speed of the LEON3 design is $25 \mathrm{MHz}$, which leads to a six orders of magnitude speedup, compared to the software-based scheme.

\section{Fault Analysis Evaluation}

To evaluate our solution for resiliency analysis, we conducted a study on our CMP router, by exposing it to several types of fault while running SPEC2000 testbenches.

Fault Effects per Fault Model - The graph in Figure 6 shows the percentage of injected faults that caused a failure, grouped by fault model. The fault injection experiments were run on the CMP router stimulated with communication traces of several SPEC CPU2000 benchmarks and a synthetic hightraffic communication trace (hi_util). We observe that the effects of the injected faults on the design vary for different fault models. Specifically, fault models related to permanent silicon failures (i.e., stuck-at, stuck-open, and bridge) have more adverse effects on the design, and $70-80 \%$ of them cause errors observable at the primary outputs of the design. On the other hand, the path delay fault model has less adverse effects, and on average only $40 \%$ of these faults manifest. Finally, the SEU faults have the least impact on the correct functionality of the design and on average less than $10 \%$ of them cause an error.

Failure Observation Latency - The graph in Figure 7 shows the average latency of an injected fault to propagate an error to the primary outputs of the design. The results shown are for different fault models for the CMP router and the LEON3 system-on-chip. The failure observation latency is a very important metric when assessing the resiliency of a design because it provides insight on whether specific error detection and recovery techniques can provide a successful recovery from the fault's effects. We noticed that the failure observation latency varies depending on the fault model. Specifically, we observed that, for the CMP router, the injected path-delay faults have the highest failure manifestation latency, while fault models associated with permanent failure mechanisms usually have similar failure manifestation latencies. Furthermore, we noticed that the error manifestation latency for SEU faults is very small. When this observation is combined with the results of the previous experiment, we concluded that SEU transient faults either cause an error in the design immediately after they occur, or they do not cause an error at all, as would be expected due to their transient nature.

We also noticed that the measured failure-observation latencies for the LEON3 system-on-chip are orders of magnitude larger than the ones observed for the CMP router. This difference stems from the higher complexity of the LEON3 system-on-chip, which in turn leads to more cycles 


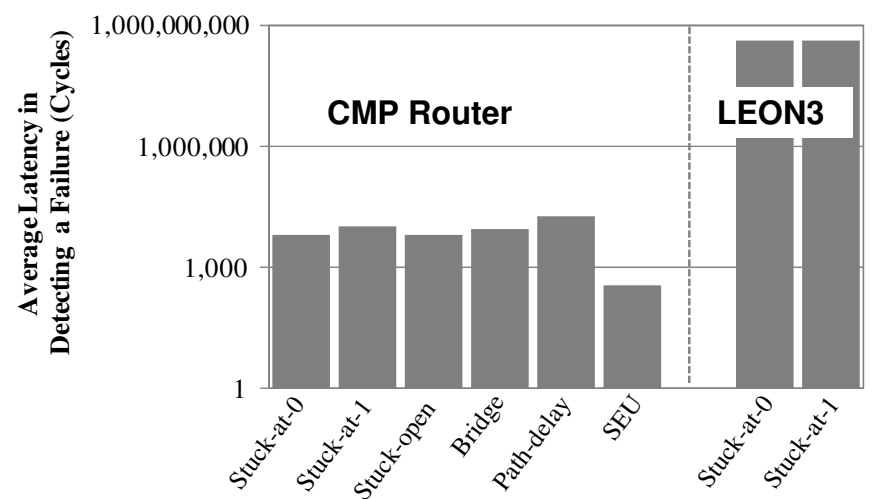

Fig. 7. Failure Detection Latency: Failure observation latency at the design's primary outputs.

required for a fault to propagate to the design's output (the output of the running application). To provide more insights on this aspect, the graph of Figure 8 shows the cumulative distribution of the injected faults over the failureobservation latency in clock cycles. Note that more than half of the injected faults propagate a failure to the application output almost immediately, but the remaining ones require billions of cycles for the failure to manifest. This observation supports the argument that if a fault hits a critical part of the design, then its effect are immediate. On the other hand, if it hits a less critical/exercised part of the design, then its effects are largely delayed.

\section{CONCLUSiOnS \& Future Work}

In this paper we presented CrashTest, a novel FGPAbased resiliency analysis framework capable of automatically orchestrating a fault injection and analysis campaign on the gate-level netlist of the design. To accelerate the fault injection process, multiple faults are injected into the design simultaneously by instrumenting the netlist with fault injection logic through gate-level logic transformations. The framework supports an extensive collection of fault models ranging from transient faults to silicon defects, and it can easily be upgraded with new fault models. We evaluated our framework on an FPGA-based platform, and found that hardware emulation can accelerate the fault simulation and analysis process by 16-90x for simple designs and six orders of magnitude for a more complex system-on-chip design, when compared to an equivalent software-based simulator.

The work presented here is the first step toward the implementation of a widely usable resiliency analysis infrastructure on an FPGA-based emulation platform [8]. We are currently working on porting the existing infrastructure to the BEE2/RAMP platform [8, 22], thereby making it widely available to researchers that are migrating to this platform. The BEE2 platform will enable the analysis of even more complex designs, and will further improve the fault simulation speed and analysis quality since more resources will be available for the fault injection instrumentation logic and the fault analysis process.

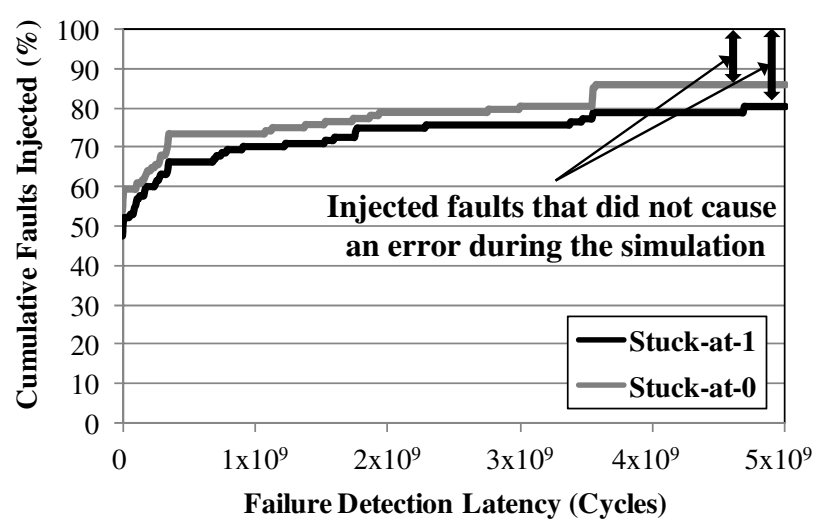

Fig. 8. Application-level Detection Latency: Latency (in cycles) for a stuck-at fault to propagate to the application outputs in the LEON3 SoC.

\section{ACKNOWLEDGMENTS}

We acknowledge the support of the Gigascale Systems Research Center (GSRC), National Science Foundation and Semiconductor Research Corporation.

\section{REFERENCES}

[1] Xilinx University Program: Xilinx XUP Virtex-II Pro Development System. http://www.xilinx.com/univ/xupv2p.html, 2005.

[2] T. M. Austin. DIVA: A reliable substrate for deep submicron microarchitecture design. In MICRO-32, 1999.

[3] J. E. Bartlett et al. Organizational research: Determining appropriate sample size in survey research. Information Technology, Learning, and Performance Journal, 19(1):43-50, 2001.

[4] S. Borkar. Microarchitecture and design challenges for gigascale integration. In MICRO, Keynote Presentation, 2004.

[5] S. Borkar et al. Design and reliability challenges in nanometer technologies. In DAC-41, 2004.

[6] F. A. Bower et al. A mechanism for online diagnosis of hard faults in microprocessors. In MICRO, 2005.

[7] M. L. Bushnell et al. Essentials of Electronic Testing for Digital, Memory and Mixed-Signal VLSI Circuits. Kluwer Academic Publishers, Boston, 2000.

[8] C. Chang et al. BEE2: A high-end reconfigurable computing system. IEEE Design \& Test of Computers, 22(2), 2005.

[9] P. Civera et al. FPGA-based fault injection techniques for fast evaluation of fault tolerance in VLSI circuits. Lecture Notes in Computer Science, 2147, 2001.

[10] J. Gaisler. A portable and fault-tolerant microprocessor based on the SPARC V8 architecture. In DSN, pages 409-415, 2002.

[11] R. Guo et al. Evaluation of test metrics: Stuck-at, bridge coverage estimate and gate exhaustive. In VTS, 2006.

[12] G. A. Kanawati et al. FERRARI: A flexible software-based fault and error injection system. IEEE Trans. Computers, 44(2):248-260, 1995.

[13] Li-Shiuan Peh. Flow Control and Micro-Architectural Mechanisms for Extending the Performance of Interconnection Networks. PhD thesis, Stanford, 2001.

[14] C. López-Ongil et al. An autonomous FPGA-based emulation system for fast fault tolerant evaluation. In FPL, 2005.

[15] C. McNairy and R. Bhatia. Montecito: A dual-core, dual-thread Itanium processor. IEEE Micro, 25(2):10-20, 2005.

[16] Mei-Chen et al. Fault injection techniques and tools. IEEE Computer, 30(4):75-82, 1997.

[17] G. P. Saggese et al. Microprocessor sensitivity to failures: Control vs execution and combinational vs sequential logic. In DSN, 2005.

[18] K. Sankaralingam et al. Exploiting ILP, DLP, and TLP using polymorphism in the TRIPS architecture. In ISCA, 2003.

[19] D. P. Siewiorek and R. S. Swarz. Reliable computer systems: Design and evaluation, 3rd edition. AK Peters, Ltd, 1998.

[20] J. Srinivasan et al. The impact of technology scaling on lifetime reliability. In $D S N-34$, pages 177-186, 2004.

[21] N. J. Wang et al. Characterizing the effects of transient faults on a high-performance processor pipeline. In DSN, 2004.

[22] J. Wawrzynek et al. RAMP: A research accelerator for multiple processors. Technical report, Berkeley, 2006. 